\title{
One anastomosis gastric bypass and esojejunostomy in rats: surgical techniques
}

\author{
Leila M'Harzi ${ }^{1,2}$, Matthieu Bruzzi ${ }^{1,2,3}$, Jean-Marc Chevallier ${ }^{1,2,3}$, Richard Douard $^{1,2,3}$ \\ ${ }^{1}$ General and Digestive Surgery Unit, Georges Pompidou, AP-HP University Hospital, Paris 75908, France. \\ 2INSERM 970, Équipe 2, PARCC, HEGP, Paris 75015, France. \\ ${ }^{3}$ Paris Descartes Faculty of Medicine, Paris 75006, France.
}

Correspondence to: Dr. Matthieu Bruzzi, Service de chirurgie digestive, Hôpital européen Georges Pompidou, 20 Rue Leblanc, Paris 75908, France. E-mail: matthieu.bruzzi@aphp.fr

How to cite this article: M'Harzi L, Bruzzi M, Chevallier JM, Douard R. One anastomosis gastric bypass and esojejunostomy in rats: surgical techniques. Mini-invasive Surg 2019;3:27. http://dx.doi.org/10.20517/2574-1225.2019.22

Received: 16 Jul 2019 First Decision: 27 Aug 2019 Revised: 12 Sep 2019 Accepted: 17 Sep 2019 Published: 23 Sep 2019

Science Editor: Wah Yang Copy Editor: Cai-Hong Wang Production Editor: Tian Zhang

\begin{abstract}
One anastomosis gastric bypass (OAGB) is a popular bariatric procedure, but controversies remain regarding biliary reflux and the potential risk of cancer. Esophagojejunostomy (EJ) in rats is a validated and reproducible model for the development of metaplasia [Barett's esophagus (BE)] and esophageal adenocarcinoma (EA) with a minimal exposure of 12 to 20 weeks. We are analyzing the risks of BE and EA in an OAGB rat model and comparing these with the EJ rat model. The purpose of this study is to describe our OAGB and EJ techniques in rats that we used to evaluate biliary reflux and share our experience with scientists and the bariatric community. These operations are short and simple procedures with acceptable morbidity.
\end{abstract}

Keywords: Mini-gastric bypass, biliary reflux, cancer

\section{INTRODUCTION}

One anastomosis gastric bypass (OAGB), or mini-gastric bypass, is a popular bariatric surgery procedure in humans ${ }^{[1-3]}$, but controversies remain regarding the risks of biliary reflux and potential complications as metaplasia, dysplasia and cancer ${ }^{[4]}$.

No studies on the risks of these for humans are currently available and research, including experimental studies, is needed to help answer this important question ${ }^{[4,5]}$. The risk of developing metaplasia [Barett's esophagus (BE)] or esophageal adenocarcinoma (EA) after omega loop montage mainly exists because of

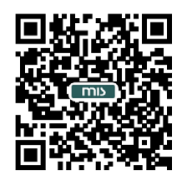




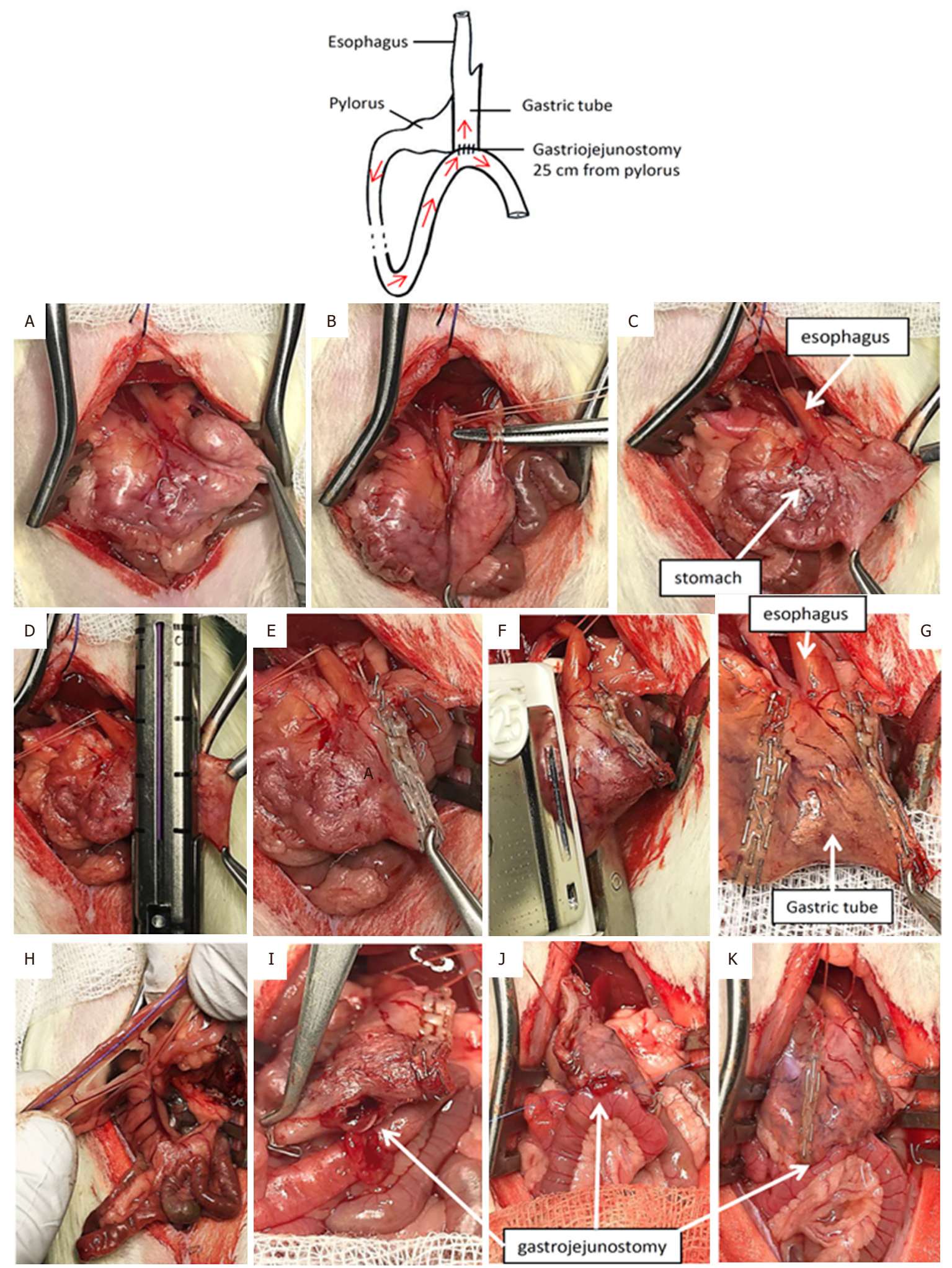

Figure 1. One-anastomosis gastric bypass

studies that analyzed surgical models in rats in which the gastro-duodenal or enteral contents were placed in direct contact with the esophageal mucosa [esophagojejunostomy $(\mathrm{EJ})]^{[6,7]}$. EJ in rats is a validated and reproducible model for the development of $\mathrm{BE}$ and $\mathrm{EA}$ with a minimal exposure of 12 to 20 weeks. 
We are analyzing and comparing the risk of metaplasia (BE) and/or EA in OAGB and EJ rat models with a follow-up of 30 weeks $^{[8]}$. The study is currently in progress but we decided to communicate the different surgical techniques that we used to share our experience with scientists and the bariatric community.

\section{METHODS}

The study complied with European Community guidelines and was approved by the ethics committee (Comité d'éthique en expérimentation animale $\left.\mathrm{n}^{\circ} 18-115\right)$. Female and male Wistar rats aged 7 weeks and weighing $250 \mathrm{~g}$ were used.

\section{Preoperative care and anesthesia}

Rats were fasted overnight before operation. Anesthesia was given by inhalation with isoflurane. Standard aseptic procedures were used throughout ${ }^{[9]}$.

\section{Materials}

Our equipment included an anesthesia workstation, Prolene 7/0 and Vicryl 3/0 sutures, a self-retaining retractor, curve dressing forceps, curved scissors, a micro needle order and needle order, scalpel handle, crile and an optical magnification system, an endo GIA $45-\mathrm{mm}$ staple gun with purple cartridge (Medtronic) and a TA-DST 30-mm-3.5-mm stapler (Covidien).

\section{Rat position and exposition}

The rat was positioned supine, feet spread apart, abdomen shaved. After laparotomy, the lateral banks were removed with a self-retaining retractor and a suture was suspended at the xyphoid.

\section{OAGB surgeries}

After retraction of the liver, the stomach was isolated [Figure 1A]. Loose gastric connections to the spleen were released along the greater curvature, and the suspensory ligament supporting the upper fundus was severed [Figure 1B]. A vicryl suture was passed behind the esophagus. Then, the gastric artery and the esophagus were separated ${ }^{[5,10]}$.

\section{The gastric pouch}

The forestomach was resected using an endo GIA 45-mm staple gun with purple cartridge (Medtronic; Figure $1 \mathrm{C}$-E). The esogastric junction was then dissected and the vascular supply isolated in this region. The stapler TA-DST $30 \mathrm{~mm}-3.5 \mathrm{~mm}$ (Covidien) position was delimited between the esophagus and the left gastric artery using the wire previously placed positioned in a parallel line with the transection line of the forestomach, and the gastric pouch created [Figures $1 \mathrm{~F}$ and $\mathrm{G}$ ].

\section{Omega limb}

The small intestines were run distally from the pylorus for $25 \mathrm{~cm}$. We recommend using a premeasured suture for this [Figure $1 \mathrm{H}$ ]. Curved scissors were used to create a 3-mm jejunostomy on the anti-mesenteric margin of bowel. The jejunum was then anastomosed to the gastric pouch $25 \mathrm{~cm}$ from the pylorus [Figure 1I].

\section{Hand-sewn gastro-jejunostomy}

The loop of bowel was identified was moved gently to the gastric pouch. A 3-to-4-mm gastrostomy was made on the gastric pouch [Figure 1I]. The anastomosis was performed manually with 7-0 Prolene running sutures. We began with the corner points on both sides of the anastomosis [Figure 1J]. When the anterior running suture was complete, we turned the gastrojejunal block and then completed the posterior running suture. The suture took serosa on the esophagus and the gastric tube. After replacing the gastrojejunal block [Figure $1 \mathrm{~K}$ ], we wrapped the anastomosis in the omentum. One milliliter of saline was then poured intraperitoneally. 


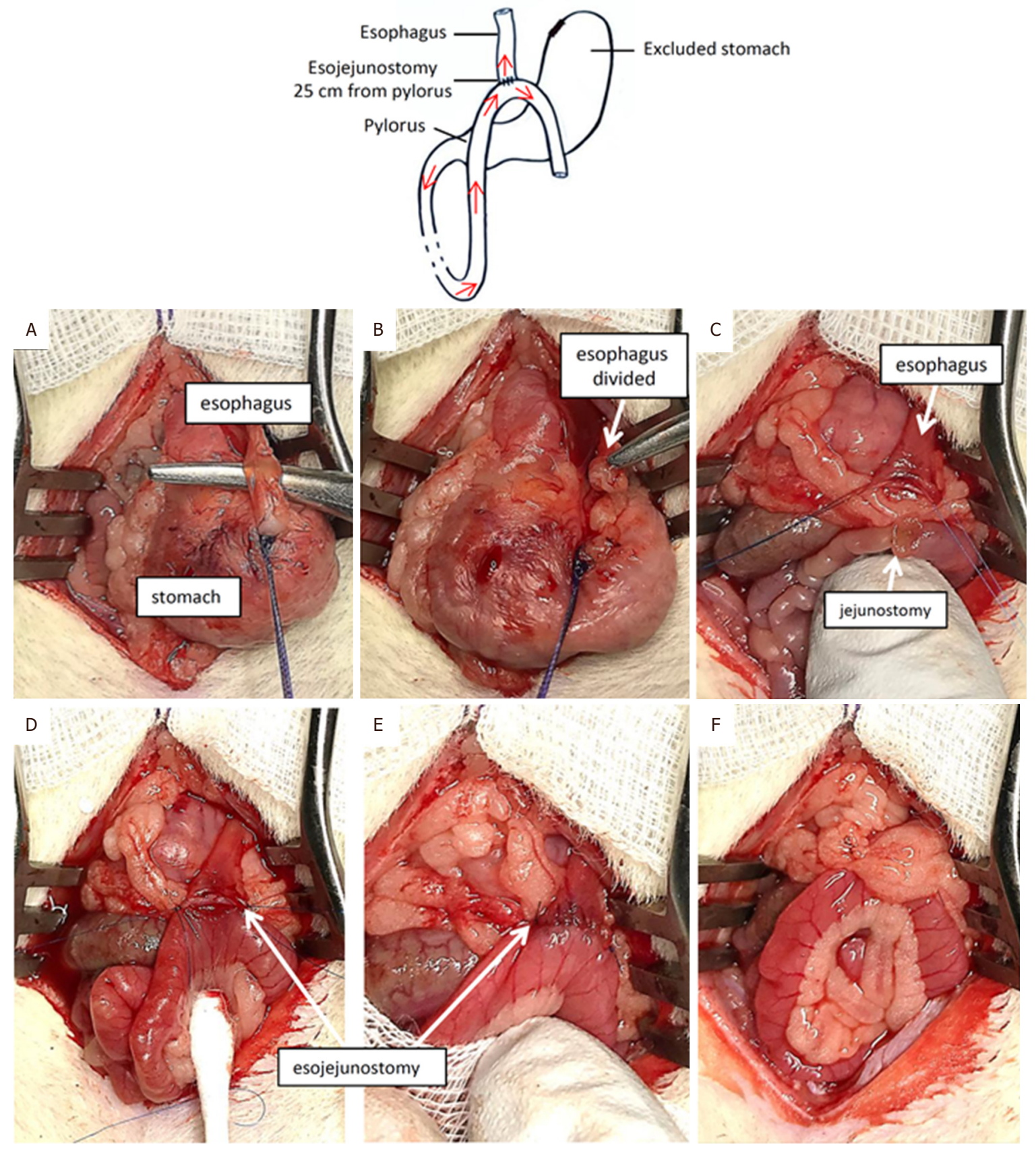

Figure 2. Esojejunostomy

\section{EJ surgeries}

The liver was retracted and the stomach isolated. Loose gastric connections to the spleen and liver were released along the greater curvature, and the suspensory ligament supporting the upper fundus was severed. A vicryl wire was passed behind the esophagus.

Section of the esophagus

The wire behind the esophagus was placed at the base of this one. It was tied and pulled down. The esophagus was cut with a scalpel, as close to the esogastric junction as possible [Figure 2A and B]. 
Before it was retracted, two Prolene 7/0 tractors were placed on both side of the esophagus, taking care to remove mucosa and serosa (total points). The knot of the stomach was reinforced with a running suture completed with Prolene 7.0 [Figure 2C].

\section{Omega limb}

The small intestines were run distally from the pylorus for $25 \mathrm{~cm}$. We recommend using a premeasured wire (as described in OAGB surgeries, Figure $1 \mathrm{H}$ ). Curved scissors were used to create a 3-mm jejunostomy on the anti-mesenteric margin of the bowel [Figure $2 \mathrm{C}$ ].

\section{Hand-sewn EJ}

The loop of the bowel was identified and moved gently to the esophagus in a precolic position [Figure 2D]. Anastomosis was performed manually using 7-0 Prolene running sutures. We started with the corner points on both sides of the anastomosis [Figure 2E]. When the anterior running suture was complete, we turned the esojejunal block and completed the posterior running suture. There are approximately 5 to 6 passes for each running suture. The suture took mucosa and serosa on the esophagus and only serosa on the jejunum.

After replacing the esojejunal block, we wrapped the anastomosis in the omentum [Figure $2 \mathrm{~F}]$. One milliliter of saline was poured intraperitoneally.

\section{RESULTS AND DISCUSSION}

\section{Postoperative care and mortality}

OAGB and EJ rats were maintained without food for $24 \mathrm{~h}$ after surgery. They received subcutaneous injections of $10 \mathrm{~mL}$ Bionolyte G5 (Baxter) on the first postoperative day (POD). From POD 2 to POD 3 , they had access to a liquid diet ad libitum. Free access to a normal diet was allowed from POD 4. Pain and distress were carefully monitored twice a day with scoring. Rats showing signs of pain or not eating were maintained on antalgics and euthanized if there was no improvement after $24 \mathrm{~h}$. In our experience, major morbidity in OAGB rat surgeries was due to anastomotic leakage and gastric tube necrosis and major morbidity in EJ surgeries was due to anastomotic stenosis. Gastric necrosis was due to an inadequate stapling of the left gastric artery at the level of the lesser curvature in our first surgeries. With increased experience, we improved the dissection of this artery to decrease these ischemic complications.

We operated on 40 rats: 16 with EJ surgery, 16 with OAGB, and 8 with sham surgery (laparotomy without any digestive suture). Of the $16 \mathrm{EJ}$ rats, acute mortality occurred in four (25\%) on mean postoperative day 6. This was due to anastomotic stenosis in all cases.

To prevent stenosis, we recommend doing the anastomosis carefully with very tiny passages on the esophageal mucosa and the jejunal serous. Second, the running suture must be tightened with low pressure. Of the 16 OAGB rats, we had 3 deaths (19\%) on mean postoperative day 4 . Mortality was due to anastomotic leakage in one rat and gastric pouch necrosis followed by peritonitis in two. To prevent these ischemic complications, we recommend carefully preserving the left gastric artery during TA stapling. In fact, this artery must be well dissected and isolated to avoid its stapling during the confection of the right side on the gastric tube.

\section{Risk of cancer after OAGB and the need for experimental studies}

Analysis of the prevalence of cancer and BE after EJ surgeries must be performed after sufficient esophageal exposure to bile and enteral juices, at least 12 to 20 weeks $^{[6,7]}$. In our experience of OAGB surgeries, no dysplasia, metaplasia or cancer were observed after a 16-week follow-up ${ }^{[5]}$. The lack of follow-up and control 
group prompts us to now evaluate OAGB and its consequences using 30-week and 50-week follow-ups ${ }^{[8]}$ and compare these results to EJ surgeries (control group).

On the day of sacrifices, histology and molecular analysis on esophageal and gastric mucosae can be performed to analyze the prevalence of BE and cancer ${ }^{[5]}$.

Concerning the esophagus, the absence of metaplasia, dysplasia or cancer in the OAGB group compared with the EJ group will suggest confer a real protective effect of the long gastric tube used in the OAGB model. Conversely, the presence of precancerous or cancerous lesions in the esogastric blocs of OAGB rats gives additional arguments to detractors of the technique to decrease its diffusion.

This short communication is a simple contribution for researchers interested in the topic of biliary reflux after OAGB because there is an urgent need to answer this specific issue, especially for patients who have already undergone surgery.

\section{DECLARATIONS}

\section{Acknowledgments}

We thank the assistance of INSERM U970, ERI in the study.

\section{Authors' contributions}

Project development: Bruzzi M, Chevallier JM, Douard R

Surgery and data Collection: M’Harzi L, Bruzzi M

Wrote the manuscript: M'Harzi L, Bruzzi M

Comment: Chevallier JM, Douard R

\section{Availability of data and materials}

Not applicable.

\section{Financial support and sponsorship}

None.

\section{Conflicts of interest}

All authors declared that there are no conflicts of interest.

\section{Ethical approval and consent to participate}

All applicable institutional and/or national guidelines for the care and use of animals were followed. All experiments were performed in compliance with the European Community guidelines and approved by the Institutional Animal Care and Use Committee.

\section{Consent for publication}

Not applicable.

\section{Copyright}

(c) The Author(s) 2019.

\section{REFERENCES}

1. Deitel M. Letter to the editor: bariatric surgery worldwide 2013 reveals a rise in mini-gastric bypass. Obes Surg 2015;25:2166-8.

2. Mahawar KK, Kumar P, Carr WR, Jennings N, Schroeder N, et al. Current status of mini-gastric bypass. J Minim Access Surg 2016;12:305-10.

3. Rutledge R. The mini-gastric bypass: experience with the first 1,274 cases. Obes Surg 2001;11:276-80. 
4. Bruzzi M, Chevallier JM, Czernichow S. One-Anastomosis gastric bypass: why biliary reflux remains controversial? Obes Surg 2017;27:545-7.

5. Bruzzi M, Duboc H, Gronnier C, Rainteau D, Couvelard A, et al. Long-term evaluation of biliary reflux after experimental oneanastomosis gastric bypass in rats. Obes Surg 2017;27:1119-22.

6. Greene CL, Worrell SG, DeMeester TR. Rat reflux model of esophageal cancer and its implication in human disease. 2015;262:910-24.

7. Gronnier C, Bruyere E, Piessen G, Briez N, Bot J, et al. Operatively induced chronic reflux in rats: a suitable model for studying esophageal carcinogenesis? Surg 2013;154:955-67.

8. Quinn R. Comparing rat's to human's age: How old is my rat in people years? Nutrition 2005;21:775-7.

9. Pritchett-Corning KR, Luo Y, Mulder GB, White WJ. Principles of rodent surgery for the new surgeon. J Vis Exp 2011; doi: $10.3791 / 2586$

10. Cavin JB, Couvelard A, Lebtahi R, Ducroc R, Arapis K, et al. Differences in alimentary glucose absorption and intestinal disposal of blood glucose after Roux-en-Y gastric bypass vs sleeve gastrectomy. Gastroenterology 2016;150:454-64. 